\section{latrogenic intestinal obstruction: a rare complication of capsule endoscopy in a patient with familial adenomatous polyposis}

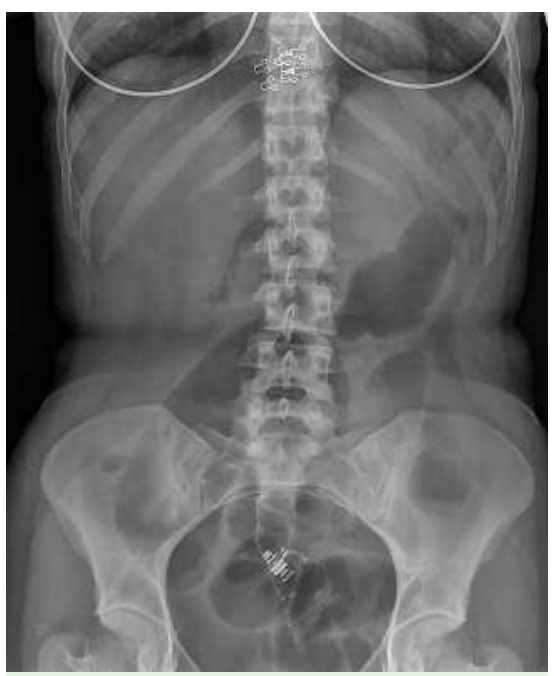

Fig. 1 Abdominal radiograph showing the capsule in the area of the anastomosis.

A 24-year-old woman with a history of an epidermoid cyst of the knee (resected at the age of 15) and a gluteal epidermal cyst (resected at the age of 23) was referred to our service. She underwent a complete colon examination because she was suspected to have familial adenomatous polyposis (FAP). Colonoscopy showed several sessile polyps along the colon. Multiple polypeptomy was performed and histopathological examination revealed these lesions to be tubular adenomas with mild dysplasia.

She went into a periodic endoscopic surveillance program until panproctocolectomy and an ileal J-pouch reconstruction were completed. Multiple adenomatous polyps with mild dysplasia were found on examining the biopsies. The majority of the polyps could not be removed, and so she was started on celecoxib-based therapy. A complete response to this treatment was achieved after 6 months of treatment, and this was confirmed by endoscopy.

She was referred to the Genetic Counselling Unit and her work-up included ocular fundus examination and a bone study, which were normal. Analysis of the APC gene in peripheral blood cells demonstrated a pathogenic mutation in exon 15. This mutation consists of a four-base deletion of CATT at codon 931. Capsule

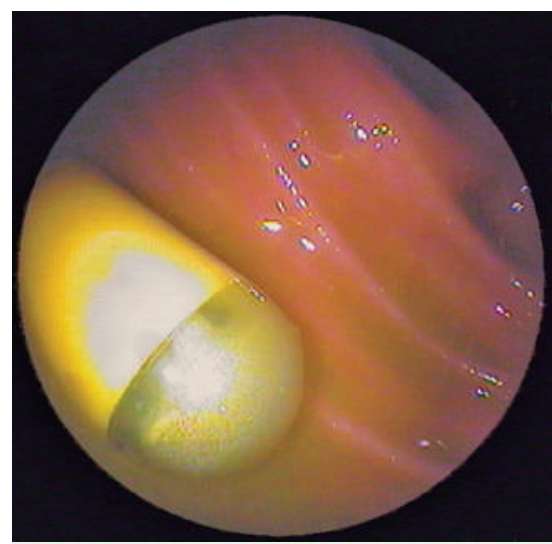

Fig. 2 Endoscopic image showing the retained capsule in the ileal reservoir, joined to one of the surgical staples.

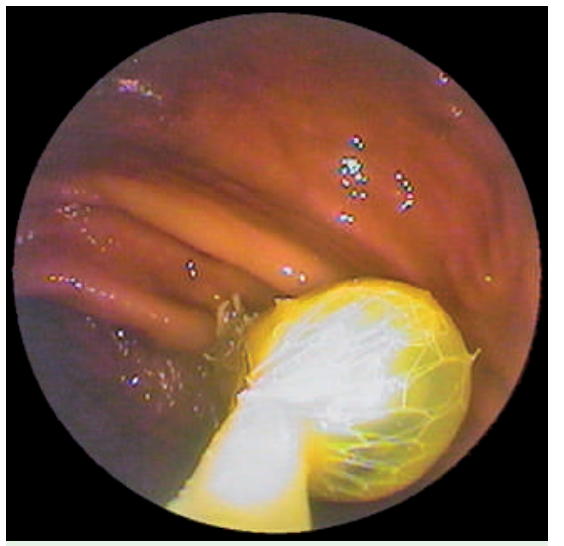

Fig. 3 The capsule was recovered endoscopically, relieving the intestinal obstruction.

endoscopy reported no polyps or mucosal alterations along the small bowel.

One year later, she required hospitalization because of intestinal pseudo-obstruction. Abdominal radiography and endoscopy showed that the capsule had been retained in the reservoir, below the intestinal anastomosis and joined to one of the surgical staples ( $\mathbf{F i g . 1 , 2}$ ). The capsule was successfully recovered by endoscopy and the intestinal obstruction was relieved ( $\bullet$ Fig. 3 ).

FAP is a hereditary colorectal syndrome with a dominant transmission and 100\% penetrance [1]. However, in $20 \%$ of cases there is no family history and the condition results from a de novo mutation, as in the present case. Recently, clinical trials have demonstrated that capsule endoscopy is a highly sensitive technique for detecting small-bowel pathology [2]. The technique has been studied mainly in inflammatory intestinal disease [3], and its application in the surveillance of patients with FAP has received relatively little attention. The detection of ileal polyps in patients with FAP could have an impact on their management, however, and complications, such as retention of the capsule, are exceptional.

Although capsule endoscopy provides a diagnosis of some potentially treatable diseases (by surgery, polypectomy, or systemic therapy) [4], we recommend that this procedure is used carefully.

\section{Endoscopy_UCTN_Code_CPL_1AI_2AB}

\section{P. Pérez-Segura ${ }^{1}$, I. Siso ${ }^{2}$, R. Luque ${ }^{3}$,} H. Olivera ${ }^{1}$, S. Díaz ${ }^{4}$, J. Mayol ${ }^{5}$,

\section{Bueno ${ }^{1}$, J. Puente ${ }^{1}$, E. Díaz-Rubio}

${ }^{1}$ Medical Oncology Department (Genetic Counselling Unit), Hospital Clínico San Carlos, Madrid, Spain

2 Medical Oncology Department, Hospital General “Gregorio Marañón”, Madrid, Spain

${ }^{3}$ Medical Oncology Department, Hospital Carlos Haya, Málaga, Spain

4 Endoscopy Department, Hospital Clínico San Carlos, Madrid, Spain

5 Gastrointestinal Surgery Department, Hospital Clínico San Carlos, Madrid, Spain 
References

1 Jo WS, Chung DC. Genetics of hereditary colorectal cancer. Semin Oncol 2005; 32:

$11-23$

2 Schulmann $K$, Hollerbach $S$, Kraus $K$ et al. Feasibility and diagnostic utility of video capsule endoscopy for the detection of small bowel polyps in patients with hereditary polyposis syndromes. Am J Gastroenterol 2005; 100: $27-37$

3 Herrerias JM, Caunedo A, Rodríguez-Tellez M et al. Capsule endoscopy in patients with suspected Crohn's disease and negative endoscopy. Endoscopy 2003; 35: 564-568

4 Eliakim R, Fisher D, Suissa A et al. Wireless capsule video endoscopy is a superior diagnostic tool in comparison to barium followthrough and computerized tomography in patients with suspected Crohn's disease. Eur J Gastroenterol Hepatol 2003; 15: $363-367$
Bibliography

DOI 10.1055/s-2007-966595

Endoscopy 2007; 39: E298-E299

(c) Georg Thieme Verlag KG Stuttgart · New York .

ISSN 0013-726X

Corresponding author

\section{P. Pérez-Segura, MD}

Medical Oncology Department

(Genetic Counselling Unit)

Hospital Clínico San Carlos

C/ Prof. Martín Lagos s/n

28040 Madrid

Spain

Fax: +34-913303544

perezsegura@yahoo.com 